\title{
NON-INTEGRABILITY OF A WEAKLY INTEGRABLE HAMILTONIAN SYSTEM
}

\author{
Giuseppe Pucacco* \\ Dipartimento di Fisica - Università di Roma "Tor Vergata" \\ and \\ INFN - Sezione di Roma II \\ Kjell Rosquist ${ }^{\dagger}$ \\ Department of Physics - Stockholm University
}

\begin{abstract}
The geometric approach to mechanics based on the Jacobi metric allows to easily construct natural mechanical systems which are integrable (actually separable) at a fixed value of the energy. The aim of the present paper is to investigate the dynamics of a simple prototype system outside the zero-energy hypersurface. We find that the general situation is that in which integrability is not preserved at arbitrary values of the energy. The structure of the Hamiltonian in the separating coordinates at zero energy allows a perturbation treatment of this system at energies slightly different from zero, by which we obtain an analytical proof of non-integrability.
\end{abstract}

\footnotetext{
*e-mail: pucacco@roma2.infn.it
}

†e-mail: kr@physto.se 


\section{Introduction}

The property of a Hamiltonian system to be integrable may happen to be satisfied only at a fixed value of the energy. In this case we can speak of weak integrability and refer to the phase-space functions which are conserved only in correspondence of those given energy values as weak invariants. From this point of view, standard integrability, with invariants which are conserved functions at arbitrary energies, can be referred to as strong integrability.

Weak invariants, also called configurational invariants, have been discussed by Hall (1983) and by Sarlet, Leach and Cantrijn (1985). Hietarinta (1987), in his account of the direct methods for the search of the second invariant, also provides a review of all the known 2-dimensional systems admitting one or more configurational invariants. In the present paper we use the approach of Rosquist and Pucacco (1995), where quadratic invariants at arbitrary and fixed energy for 2-dimensional systems were treated in a unified way. As shown in that paper, the integrability condition for quadratic invariants, corresponding to secondrank Killing tensors of the conformal Riemannian geometry with Jacobi metric, involves an arbitrary analytic function $S(z)$. For invariants at arbitrary energy, the function $S(z)$ is a second degree polynomial with real second derivative and the integrability condition then reduces to the classical Darboux's condition for quadratic invariants at arbitrary energy (Darboux, 1901; Whittaker, 1937). The possibility of searching for linear and quadratic invariants at fixed energy was also addressed and some examples of systems admitting a second quadratic invariant at zero energy were provided.

Generalizing the approach, Karlovini and Rosquist (2000) have discussed the existence of invariants cubic in the momenta at both fixed and arbitrary energy. Besides giving a list of all known systems admitting a cubic strong invariant, they find a superintegrable system admitting a cubic configurational invariant related to an energy dependent linear invariant. In Karlovini, Pucacco, Rosquist and Samuelson (2002) we discuss the case of quartic invariants associated with the existence of fourth-rank Killing tensors. The results obtained in this geometric framework are in agreement with the direct method when the class of potentials examined is the same, as can be seen comparing the cases listed in the above papers with those appearing in Nakagawa and Yoshida (2001).

The set of weakly integrable systems is very large but, on the other hand, they are probably of limited usefulness in physical applications. One may wonder however if the knowledge of a weak invariant can provide information about the global dynamical behaviour of the system. One possibility is to investigate the existence of integrable systems with a higher-order strong invariant related to the weak one (e.g. linear in the momenta, as in one example given in Karlovini and Rosquist, 2000). The aim of the present paper is instead to explore the phase-space structure of the system at energies different from that assuring weak integrability in the general situation in which the system is non-integrable. In one class of systems, we show how the property of weak inte-

grability leads to a better understanding of the dynamics of generic systems. In particular, the setting in which the geometric approach casts the problem allows 
to apply powerful tools of analytical mechanics to prove the non-integrability of the dynamical system.

The plan of the paper is as follows: in section 2 we recall conditions for weak and strong integrability of a two-dimensional natural Hamiltonian system limiting the application to the existence of a second invariant quadratic in the momenta; in section 3 we present a class of weakly integrable systems which have a quasi-regular behavior at near-zero energy and for which we give a Poincaré type argument of non integrability out of the zero energy surface; section 4 contains the conclusions.

\section{Integrability at fixed energy}

The technique devised and applied in Rosquist and Pucacco (1995, RP here and in the following), allows to find the conditions such that a 2-dimensional natural Hamiltonian system admits a second invariant quadratic in the momenta. In this section we briefly recall these results that are at the basis of the applications of the following sections.

\subsection{Weak quadratic invariants}

We are interested in the classical 2-dimensional systems with Hamiltonian function

$$
\mathcal{H}=\frac{1}{2}\left(p_{x}^{2}+p_{y}^{2}\right)+V(x, y) .
$$

Since the Hamiltonian is time-independent, energy is conserved and motion takes place on the hypersurface

$$
\mathcal{H}\left(p_{x}, p_{y}, x, y\right)=E .
$$

The approach of RP amounts to find a conformal transformation to new coordinates $X, Y$ defined by

$$
z=F(w), \quad z=x+i y, \quad w=X+i Y,
$$

generated by an arbitrary analytic function $S(z)$ via the relation

$$
F^{\prime}(w(z))=\sqrt{S(z)} .
$$

The transformation (3) naturally induces a canonical point transformation that gives the new Hamiltonian

$$
\mathcal{H}_{S}=\frac{\frac{1}{2}\left(p_{X}^{2}+p_{Y}^{2}\right)}{|S(X, Y)|}+V(X, Y) .
$$

where it appears the conformal factor

$$
|S(X, Y)|=\sqrt{S(w) \bar{S}(\bar{w})}=F^{\prime}(w) \bar{F}^{\prime}(\bar{w}) .
$$


One can then show that, if the function

$$
G=E-V,
$$

the so-called "Jacobi" potential, can be expressed, in the new coordinates $X, Y$, in the form

$$
G(X, Y)=\frac{A(X ; E)+B(Y ; E)}{|S(X, Y)|},
$$

where $A$ and $B$ are arbitrary functions of their arguments, the quadratic function

$$
I_{0}\left(p_{X}, p_{Y}, X, Y\right)=\frac{1}{2}\left(p_{X}^{2}-p_{Y}^{2}\right)-A(X)+B(Y),
$$

commutes with the Hamiltonian (5) on the surface

$$
\mathcal{H}\left(p_{x}, p_{y}, x, y\right)=0 \text {. }
$$

We therefore speak of a weak invariant of the standard Hamiltonian system, in contraposition with the usual notion of strong invariant by which we mean a phase-space function which is conserved at arbitrary values of the energy.

The origin of the phenomenon of weak integrability in the present context is due to the geometrization of the dynamics via the Jacobi-Maupertuis variational principle. For a detailed account of the Jacobi geometrization we refer to standard textbooks (Abraham and Marsden, 1978; Arnold, 1978; Lanczos, 1986) and to RP where, in particular, the link is deepened between quadratic invariants and second-rank Killing tensors which is at the basis of this approach. Here we briefly recall that to the Jacobi potential (7) pertains a family of conformal Riemannian metrics parametrized by the value of the energy. Each energy specifies a geodesic flow on a Riemannian manifold, whose projections coincide with the orbits of the system defined by the standard Hamiltonian up to a time reparametrization. The solution of the Killing tensor equations provides an invariant along the given flow at a given value of the energy parameter (in particular at zero energy). It is only the additional requirement that the solution of Killing equations be independent of the energy that leads to integrability at arbitrary energy and poses a further constraint on the generating function $S(z)$. If this condition is satisfied, the function (9) commutes with the Hamiltonian (5) on the surface (2), $\forall E$. One can clearly also extend the investigation of higher rank Killing tensors or more general functional forms of the second invariant and to the corresponding analysis of the conditions for strong integrability.

\subsection{Strong quadratic invariants}

In RP it has been shown that, to get quadratic invariants at arbitrary energy, the function $S(z)$ must satisfy the condition

$$
\operatorname{Im}\left\{S^{\prime \prime}(z)\right\}=0,
$$

that is it must be a second degree polynomial with real second derivative. Actually, we can show that condition (11) not only implies strong integrability, but also assures the separation of variables. We have in fact the following 
Theorem 1: Given the conformal transformation

$$
d z=F^{\prime}(w) d w=\sqrt{S(z(w))} d w,
$$

condition necessary and sufficient to have

$$
|S(X, Y)|=A_{S}(X)+B_{S}(Y),
$$

where $A_{S}$ and $B_{S}$ are real analytic functions of a single variable, is that (11) is true.

Proof: Let us prove that (13) implies (11). Functional form (13) is equivalent to say that the function $|S|$ must satisfy the differential equation

$$
|S|_{, X Y}=0 .
$$

But

$$
\begin{aligned}
|S|_{, X Y} & =i\left[|S|_{, w w}-|\bar{S}|_{, \bar{w} \bar{w}}\right]=i\left[\sqrt{S}\left(\sqrt{S}(\sqrt{S \bar{S}})_{, z}\right)_{, z}-\sqrt{\bar{S}}\left(\sqrt{\bar{S}}(\sqrt{S \bar{S}})_{, \bar{z}}\right) 1, \bar{z}\right] \\
& =i|S|\left(S_{, z z}-\bar{S}_{, \bar{z} \bar{z}}\right)=-2|S| \operatorname{Im}\left\{S^{\prime \prime}\right\}
\end{aligned}
$$

Since $|S|$ is a non-null positive function everywhere, the implication is evident. On the contrary, (11) is equal to

$$
S=a z^{2}+\beta z+\gamma,
$$

with $a$ real and $\beta, \gamma$ complex. This implies (13) as can be verified by performing the coordinate transformation (3), with

$$
w=F^{-1}(z)=\int \frac{d z}{\sqrt{a z^{2}+\beta z+\gamma}},
$$

where (17) has been used in defining the generating function via (4). Q.E.D.

In RP, Sect. 4, the explicit forms of functions $A_{S}$ and $B_{S}$ are given. They can be of the four possible types corresponding to separability in Cartesian, polar, parabolic and elliptic coordinates. In this way, if we write the two arbitrary functions appearing in (8) as

$$
\begin{aligned}
& A(X ; E)=E A_{S}(X)-f(X), \\
& B(Y ; E)=E B_{S}(Y)-g(Y),
\end{aligned}
$$

the "true" potential takes the form

$$
V(X, Y)=\frac{f(X)+g(Y)}{|S(X, Y)|},
$$

and the Hamilton-Jacobi equation for Hamilton's characteristic function, $\mathcal{W}(X, Y)$, associated to Hamiltonian (5), takes on the explicitly separated form

$$
\frac{1}{2}\left[\left(\mathcal{W}_{, X}\right)^{2}+\left(\mathcal{W}_{, Y}\right)^{2}\right]+f(X)+g(Y)-E\left[A_{S}(X)+B_{S}(Y)\right]=0,
$$

and therefore strongly integrable two-dimensional systems with quadratic second invariants can be exhaustively classified. 


\subsection{Regularization}

Before starting the analysis of weakly integrable system, it is useful to recall the fact that the conformal transformation leads, in a natural way, to a time reparametrization of the dynamics which can be usefully exploited in the applications. This reparametrization has been used since a long time in the framework of the "regularization" of singular differential equations, especially in the applications in celestial mechanics (see, e.g. Levi-Cìvita, 1956; Sundman, 1912). We can therefore speak of a generalized regularization approach and the null Hamiltonian introduced below can be called on the same footing the regularized Hamiltonian.

Let us suppose that we have chosen a function $S(z)$ which generates the conformal transformation which gives a system admitting a weak second invariant (9). If we denote by $\mathcal{H}_{0}$ the function

$$
\frac{1}{2}\left(p_{X}^{2}+p_{Y}^{2}\right)+f(X)+g(Y)
$$

which can be interpreted as the numerator appearing in the Hamiltonian (5) once the expression (21) is taken into account, the Poisson brackets of a generic phase-space function $J$ with $\mathcal{H}_{S}$ can be written as

$$
\left\{J, \mathcal{H}_{S}\right\}=\frac{1}{|S|}\left\{J, \mathcal{H}_{0}\right\}-\frac{\mathcal{H}_{0}}{|S|^{2}}\{J,|S|\}=\frac{\left\{J, \mathcal{H}_{0}\right\}-E\{J,|S|\}}{|S|} .
$$

On the other hand, let us introduce the null Hamiltonian

$$
\mathcal{H}_{\mathcal{N}}=\left(\mathcal{H}_{S}-E\right)|S|=\mathcal{H}_{0}-E|S|
$$

The Poisson bracket of a function with $\mathcal{H}_{\mathcal{N}}$ is given by

$$
\left\{J, \mathcal{H}_{\mathcal{N}}\right\}=\left\{J, \mathcal{H}_{0}\right\}-E\{J,|S|\}
$$

Eqs.(24) and (26) are therefore equivalent with respect to conservation of $J$. The conceptual difference relates to the fact that, whereas vanishing of eq.(26) expresses the possible conservation of $J$ in the dynamics provided by $\mathcal{H}_{\mathcal{N}}$ at zero "energy" (i.e. $\mathcal{H}_{\mathcal{N}}=0$ ), the vanishing of eq.(24) expresses the conservation of $J$ in the dynamics provided by $\mathcal{H}_{S}$ at arbitrary energy. Note that the physical energy $E$ enters into $\mathcal{H}_{\mathcal{N}}$ as an arbitrary parameter.

With the null Hamiltonian, it is automatically introduced a new (regularizing) time variable $\eta$ by means of

$$
d \eta=\frac{d t}{|S|}
$$

Just as the Poisson brackets (24) express the total derivative with respect to the standard time

$$
\frac{d J}{d t}=\left\{J, \mathcal{H}_{S}\right\}
$$


the Poisson brackets (26) express the total derivative with respect to the regularizing time

$$
\frac{d J}{d \eta}=\left\{J, \mathcal{H}_{\mathcal{N}}\right\}
$$

Correspondingly, the "regularized" equations of motion are

$$
\begin{array}{ll}
\frac{d X}{d \eta}=\left\{X, \mathcal{H}_{\mathcal{N}}\right\}, & \frac{d p_{X}}{d \eta}=\left\{p_{X}, \mathcal{H}_{\mathcal{N}}\right\}, \\
\frac{d Y}{d \eta}=\left\{Y, \mathcal{H}_{\mathcal{N}}\right\}, & \frac{d p_{Y}}{d \eta}=\left\{p_{Y}, \mathcal{H}_{\mathcal{N}}\right\} .
\end{array}
$$

It is worthwhile to remark that $\mathcal{H}_{S}$ gives the same dynamics as the original physical Hamiltonian $\mathcal{H}$. Only different coordinates are used as a result of a canonical point transformation. The time reparametrization implies the relation between Poisson brackets associated with the original physical Hamiltonian and the null regularized one. Considering the phase-space function of the regularized system as $J\left(p_{X}, p_{Y}, X, Y ; E\right)$ and the corresponding phase-space function of the original system, say $I\left(p_{x}, p_{y}, x, y\right)$, obtained via the recipe

$$
\left\{p_{X}, p_{Y}, X, Y\right\} \rightarrow\left\{p_{x}, p_{y}, x, y\right\}, \quad E \rightarrow \mathcal{H}\left(p_{x}, p_{y}, x, y\right),
$$

the total time-derivative along the flow of these two functions are related by

$$
\frac{d J}{d \eta}=|S| \frac{d I}{d t}
$$

The conformal factor is never vanishing and therefore we extend considerations above on conserved quantities to functions in the original coordinate frame.

It should also be recalled that the combination of the conformal transformation (3) with the introduction of the new time variable by means of (27) can also be seen as a canonical transformation on the extended phase space where $\mathcal{H}$ and $t$ are new canonical coordinates (Tsiganov, 2000).

\subsection{Dynamics at zero energy}

The dynamics provided by the null or regularized Hamiltonian (25) is particularly simple in the separable case: the two motions in the separating coordinates $X$ and $Y$ decouple and the general motion is given by a superposition with independent arbitrary initial conditions. Clearly, the same happens in the even simpler case of a system constructed as above choosing an arbitrary conformal transformation and selecting only motions at zero energy.

In the $\eta$ time, the equations of motion (30) given by Hamiltonian (23) are simply

$$
\begin{aligned}
& \frac{d^{2} X}{d \eta^{2}}=-f^{\prime}(X), \\
& \frac{d^{2} Y}{d \eta^{2}}=-g^{\prime}(Y) .
\end{aligned}
$$


The two "energy" equations

$$
\begin{aligned}
\frac{1}{2} p_{X}^{2}+f(X) & =h_{1}, \\
\frac{1}{2} p_{Y}^{2}+g(Y) & =h_{2},
\end{aligned}
$$

with

$$
h_{1}+h_{2}=0,
$$

delimit the regions admitted to the motion by means of the inequalities

$$
\begin{aligned}
f(X) & \leq h_{1}, \\
g(Y) & \leq h_{2} .
\end{aligned}
$$

From the theory of conformal transformations (see, e.g., Markushevitch, 1983), the "isothermal" net of resulting coordinates is orthogonal (except in the finite number of isolated singularities where $F^{\prime}=0$ ). Since in the general case, the frequencies of the two motions in (30) are incommensurable, the regions admitted by the motion, with coordinates satisfying inequalities (37), are densely filled by the representative point. This happens both in the presence of rotations and librations with the two possibilities determined by the explicit form of the potential functions $A$ and $B$ and by initial conditions. This picture is the same even if we describe the dynamics with the original Hamiltonian $\mathcal{H}_{S}$. What changes is only the speed of the representative point along the trajectory.

Due to the almost trivial nature of the dynamics, we would like to know if we can exploit this simplicity to gain information about the much more complex situation of arbitrary energy. In the hypothesis of integrability at zero energy, at energies different from zero there are two possibilities: either the integrability of the system is preserved or it is broken. In the first case, a second strong invariant exists but there is no general procedure to find it. Isolated systems with second invariants which are polynomial in the momenta can be identified with the techniques described in the papers cited in the Introduction. However, the general case we expect is that in which integrability breaks down. This is the subject of the rest of the paper, where we address the following question: can we use the setting developed so far to investigate and possibly predict the non-integrability of a given system?

Before closing this section, we would however mention the fact that, due to the possibility of quite involved conformal transformations, a motion that looks trivial in the separating variables, may become complicated with many peculiar features when displayed in the physical coordinates of the original problem. Therefore, even the case with integrability limited to the hypersurface of zero energy can have direct practical applications.

\section{Systems of the class $S(z)=i z^{2}$}

We start now the analysis of 2-dimensional systems generated via general conformal transformations that do not stay in the restricted class specified by Theorem 
1. These systems are therefore integrable (actually separable) on the zero-energy surface. We have selected a set of systems for which the question posed in the previous section can be given a meaningful answer, with the criteria of the simplest non-trivial situations.

We consider the function

$$
S(z)=i z^{2} .
$$

This is the simplest polynomial which does not satisfy the constraint (11) and therefore gives a potential which is not automatically integrable at arbitrary energy.

Let us examine the coordinate transformation generated by (39). By virtue of (4), the corresponding conformal transformation is given by

$$
F(w)=\exp \left\{\frac{1+i}{\sqrt{2}} w\right\} .
$$

Using polar coordinates so that $z=x+i y=r \exp (i \vartheta)$ we get $^{1}$

$$
X=\frac{1}{\sqrt{2}}(\vartheta+\ln r), \quad Y=\frac{1}{\sqrt{2}}(\vartheta-\ln r) .
$$

The $X, Y=$ constant curves are respectively given by the two families

$$
r_{1}(\vartheta)=\exp (\sqrt{2} X-\vartheta), \quad r_{2}(\vartheta)=\exp (\sqrt{2} Y+\vartheta) .
$$

The range of $X, Y$ is from $-\infty$ to $+\infty$ so that the punctured plane (with the origin excluded) is covered with a one-to-one correspondence.

\subsection{A class of weakly integrable systems with $S(z)=i z^{2}$}

From the treatment of section 2 , it follows that the potential given by

$$
V(X, Y)=\frac{f(X)+g(Y)}{r^{2}},
$$

with the separating coordinates of (41), is integrable at zero energy for arbitrary functions $f$ and $g$. The factor $r^{-2}$ comes from eq. (6) that, from the choice of eq.(39), gives

$$
|S(X, Y)|=\mathrm{e}^{\sqrt{2}(X-Y)}=r^{2} .
$$

Due to the presence of the polar angle as explicit argument in combination with the logarithm of the radial coordinate, one must be careful in the selection of the arbitrary functions to avoid unphysical multivaluedness in the resulting potential function. A simple choice giving a smooth glueing of the sheets, resulting in a continuous and single-valued potential, is the following

$$
\begin{aligned}
& f(X)=\frac{1}{2}(C-\sin \sqrt{2} X), \\
& g(Y)=\frac{1}{2}(C-\sin \sqrt{2} Y),
\end{aligned}
$$

\footnotetext{
${ }^{1}$ Note that in RP, in equations (102-104), there is a misprint and the coordinate $r$ must be substituted with its natural logarithm.
} 
where $C$ is a real constant. Using (41), the explicit form of the potential in polar coordinates is

$$
V(r, \vartheta)=\frac{C-\sin \vartheta \cos (\ln r)}{r^{2}} .
$$

When the parameter $C$ lies in the interval

$$
0<C \leq 1,
$$

the motion in the potential (47) is bound for every value of the energy below a positive threshold, since the equipotentials are closed regular curves around the unique absolute minimum of the function $V(r, \vartheta)$,

$$
E_{\text {min }}=\min \{V(r, \vartheta)\}<0 .
$$

At the threshold energy, $E_{T}>0$, the equipotential is asymptotically open and the motion is of parabolic type. We are interested in the bound motion, so that we study the energy range

$$
E_{\text {min }}<E<E_{T} .
$$

At zero energy we can readily verify that the system is indeed integrable. Using momenta expressed in polar coordinates according to

$$
\begin{aligned}
r p_{r} & =x p_{x}+y p_{y}, \\
p_{\vartheta} & =x p_{y}-y p_{x},
\end{aligned}
$$

the second invariant has the form

$$
I_{0}\left(p_{r}, p_{\vartheta}, r, \vartheta\right)=r p_{r} p_{\vartheta}-\cos \vartheta \sin (\ln r) .
$$

It is straightforward to check that this function commutes with Hamiltonian

$$
\mathcal{H}=\frac{1}{2}\left(p_{r}^{2}+\frac{p_{\vartheta}^{2}}{r^{2}}\right)+\frac{C-\sin \vartheta \cos (\ln r)}{r^{2}}
$$

on the surface (10).

Moreover, a direct inspection allows to find the main periodic orbits. We have the marginally stable orbit

$$
r \equiv 1
$$

and the two stable periodic orbits given by

$$
\vartheta= \pm \ln r
$$




\subsection{Dynamics outside the zero energy shell}

The interesting question is now: what is the nature of the motion when the energy is different from zero? Since the system has been constructed as the simplest generalization of separable systems, it could be still integrable possibly with a more complicated second invariant. A systematic investigation of polynomial invariants of higher degree has been unsuccessful. This is clearly not enough to state non-integrability of system (54). However, we can take the other way and try to see if we can devise a test of non-integrability. Actually, it would be highly desirable to have an analytic test of non-integrability, since it can escape a numerical approach, such as the computation of the Poincaré surface, if stochastic zones are exceedingly small to be detected.

An analytic proof can be obtained by applying the Poincaré theorem (Poincaré, 1892; Whittaker, 1937) on non-existence of additional invariants in non-degenerate systems. To this end we follow a perturbative approach in which the value of the energy plays the role of the perturbation parameter, with zero energy defining the unperturbed system. We remark that this procedure can be applied to the regularized system whereas it seems very difficult to attempt it in the original variables. is

In the present instance, the null Hamiltonian (25) in the separating variables

$$
\mathcal{H}_{\mathcal{N}}=\frac{1}{2}\left(p_{X}^{2}+p_{Y}^{2}\right)-\frac{1}{2}(\sin \sqrt{2} X+\sin \sqrt{2} Y)+C-E \mathrm{e}^{\sqrt{2}(X-Y)} .
$$

With the further change of variable

$$
\begin{aligned}
& \alpha(X)=\sqrt{2} X-\frac{\pi}{2}=\vartheta+\ln r-\frac{\pi}{2} \\
& \beta(Y)=\sqrt{2} Y-\frac{\pi}{2}=\vartheta-\ln r-\frac{\pi}{2}
\end{aligned}
$$

we obtain the Hamiltonian

$$
\mathcal{H}_{P}=\frac{1}{2}\left(p_{\alpha}^{2}+p_{\beta}^{2}\right)-\cos \alpha-\cos \beta-2 E \mathrm{e}^{\alpha-\beta}=-2 C .
$$

We can now express the system in the standard form $\mathcal{H}_{P}=\mathcal{H}_{0}+\epsilon \mathcal{H}_{1}$, where an integrable "zero" order Hamiltonian is perturbed by a non-integrable first order term. In our case we have

$$
\begin{aligned}
\mathcal{H}_{0} & =\frac{1}{2}\left(p_{\alpha}^{2}+p_{\beta}^{2}\right)-\cos \alpha-\cos \beta \\
\mathcal{H}_{1} & =\mathrm{e}^{\alpha-\beta} \\
\epsilon & =-2 E
\end{aligned}
$$

It is then necessary to express this Hamiltonian as a function of the action-angle variables of the unperturbed system:

$$
\mathcal{H}_{P}=\mathcal{H}_{0}\left(J_{1}, J_{2}\right)+\epsilon \mathcal{H}_{1}\left(J_{1}, J_{2}, \theta_{1}, \theta_{2}\right) .
$$


The canonical variables satisfy the equations of motion

$$
J_{k}^{\prime}=-\frac{\partial \mathcal{H}_{P}}{\partial \theta_{k}}, \quad \theta_{k}^{\prime}=\frac{\partial \mathcal{H}_{P}}{\partial J_{k}}, \quad(k=1,2),
$$

where the prime denotes differentiation with respect to $\eta$, the new time introduced in (27). For $E=0(\epsilon=0)$, the unperturbed motion is given by:

$$
\begin{aligned}
J_{k}^{\prime}=0 & \rightarrow J_{k}=\text { const } \\
\theta_{k}^{\prime}=\frac{\partial \mathcal{H}_{0}}{\partial J_{k}}=\omega_{k}=\text { const } & \rightarrow \theta_{k}=\omega_{k} \eta+\text { const. }
\end{aligned}
$$

The energy equations (34) represent in this case the two uncoupled pendulums of the unperturbed Hamiltonian. Now we have

$$
h_{1}+h_{2}=-2 C
$$

In view of (48), we are considering only libration motions. It follows that the action variables are (see, e.g., Boccaletti and Pucacco, 1996)

$$
J_{k}=\frac{8}{\pi}\left[E\left(\kappa_{k}^{2}\right)+\left(\kappa_{k}^{2}-1\right) K\left(\kappa_{k}^{2}\right)\right]
$$

where

$$
\kappa_{k}^{2}=\frac{1}{2}\left(1+h_{k}\right), \quad 0 \leq \kappa_{k}^{2}<1
$$

and $K\left(\kappa_{k}^{2}\right)$ and $E\left(\kappa_{k}^{2}\right)$ are the complete elliptic integrals of first and second kind. $\mathcal{H}_{0}$ is implicitly expressed in terms of the actions by means of

$$
\mathcal{H}_{0}\left(J_{1}, J_{2}\right)=2\left(\kappa_{1}^{2}\left(J_{1}\right)+\kappa_{2}^{2}\left(J_{2}\right)-1\right) .
$$

Also the main periodic orbits of the unperturbed potential of Hamiltonian (60) deserve to be mentioned. The two periodic orbits

$$
\alpha=0, \quad \beta=0,
$$

are elliptic stable and coincide with the two periodic orbits in eq.(156). The two periodic orbits

$$
\alpha-\beta=0
$$

and

$$
\alpha+\beta=0
$$

are of parabolic type (that is they are marginally stable with characteristic exponents \pm 1 ). In particular (73) coincides with the orbit of eq. (55). These orbits are "resonant" since their frequencies $\omega_{1}, \omega_{2}$ are equal and are members of a family given by all the possible phase shifts $\lambda$ with

$$
0 \leq \lambda \leq \frac{2 \pi}{\omega_{1}}
$$


Orbit (73) corresponds to $\lambda=0$, whereas orbit (74) corresponds to $\lambda=\pi / \omega_{1}$. Parabolicity is just related to the existence of resonant tori in the unperturbed system. A perturbation "breaks" these tori generating isolated stable and unstable periodic orbits. In general, in non-degenerate systems we may expect the existence of a generic resonance that in the following will be taken of the form

$$
\omega_{1}=(p / q) \omega_{2},
$$

with $p$ and $q$ integers such that $p \leq q$.

\subsection{Poincaré theorem on non-existence of additional in- variants}

Poincaré method to demonstrate non-integrability of a Hamiltonian systems is based on proving that, under some genericity conditions, no analytic second invariant exist besides the Hamiltonian itself. We state the theorem as it is presented in Poincaré (1892) and Whittaker (1937). Then we show (theorem 5 below) that our system (57) comply with the requirements of Poincaré theorem, proving therefore its non-integrability and, as a consequence, non-integrability of original system (54). Moreover, by exploiting lemma 4 used in the proof of theorem 5 , we will show in the following section the existence of isolated periodic orbits and verify that their stable (or unstable) nature varies with respect to $E$.

We start with a definition. Assume that, for $J_{1}=\hat{J}_{1}$ and $J_{2}=\hat{J}_{2}$, frequencies $\hat{\omega}_{1}$ and $\hat{\omega}_{2}$ are commensurate according to relation (76). $J_{1}, J_{2}$ satisfying this condition are said to belong to the Poincaré set. For a two dimensional system, "Poincaré non-existence theorem" can be stated as follows:

Theorem 2: Let the Hamiltonian

$$
\mathcal{H}_{P}=\mathcal{H}_{0}\left(J_{1}, J_{2}\right)+\epsilon \mathcal{H}_{1}\left(J_{1}, J_{2}, \theta_{1}, \theta_{2}\right)
$$

satisfy the following hypotheses:

1. $\mathcal{H}_{0}$ is non-degenerate, that is:

$$
\operatorname{det}\left(\frac{\partial^{2} \mathcal{H}_{0}}{\partial J_{k}^{2}}\right) \neq 0
$$

2. $\mathcal{H}_{1}$ is generic, that is, defining the Fourier expansion

$$
\mathcal{H}_{1}\left(J_{1}, J_{2}, \theta_{1}, \theta_{2}\right)=\sum_{m, n=-\infty}^{+\infty} h_{m n}\left(J_{1}, J_{2}\right) \mathrm{e}^{i\left(m \theta_{1}+n \theta_{2}\right)},
$$

no coefficient $h_{m n}\left(J_{1}, J_{2}\right)$ is zero in the Poincaré set;

then, there is no analytic second invariant of the form

$$
I=\sum_{s=0}^{\infty} \epsilon^{s} I_{s}\left(J_{1}, J_{2}, \theta_{1}, \theta_{2}\right)
$$


independent of $\mathcal{H}$.

Proof: We refer to Poincaré (1892, sects. 81-83) and Whittaker (1937, sect. 165). For a more recent proof, see Giorgilli (2002, sect.3.1). Q.E.D.

To show the non existence of analytic second invariants of the form 800 is equivalent to exclude Liouville integrability. However, it must be remarked that the above result apply in the case of a regular function which is uniformly continuous in the phase-space variables and in a small range of the perturbation parameter, but the possibility cannot be excluded that for small fixed values of $\epsilon$ does a non-uniform invariant indeed exist (see, e.g., Kozlov, 1991, sect. 4.1).

We now proceed to verify that Hamiltonian (60) satisfy the conditions required to apply theorem 2. Let us check the non-degeneracy condition first.

Lemma 3: The Hamiltonian (71) is non-degenerate.

Proof: The frequencies of the unperturbed motion can be calculated as follows (see, Boccaletti and Pucacco (1996), sect. 1.16):

$$
\omega_{k}=\frac{\partial \mathcal{H}_{0}}{\partial J_{k}}=\frac{\partial}{\partial J_{k}}\left(2 \kappa_{k}^{2}\right)=4 \kappa_{k}\left(\frac{\partial J_{k}}{\partial \kappa_{k}}\right)^{-1}=\frac{\pi}{2} \frac{1}{K\left(\kappa_{k}^{2}\right)},
$$

where we have used

$$
\frac{\partial J_{k}}{\partial \kappa_{k}}=\frac{8}{\pi} \kappa_{k} K\left(\kappa_{k}^{2}\right)
$$

which comes from eq. (69) and standard properties of the elliptic integrals. With an analogous procedure we can compute the second derivative

$$
\frac{\partial^{2} \mathcal{H}_{0}}{\partial J_{k}^{2}}=\frac{\partial \kappa_{k}}{\partial J_{k}} \frac{\partial^{2} \mathcal{H}_{0}}{\partial \kappa_{k} \partial J_{k}}=4\left(\frac{\partial J_{k}}{\partial \kappa_{k}}\right)^{-1} \frac{\partial \kappa_{k}}{\partial J_{k}}=\frac{16}{\pi^{2}} \frac{1}{\kappa_{k}^{2} K^{2}\left(\kappa_{k}^{2}\right)} .
$$

We recall that $K\left(\kappa^{2}\right)$ is a positive uniformly increasing function in the interval $0 \leq \kappa^{2}<1$, such that

$$
K(0)=\frac{\pi}{2}, \quad K(1) \rightarrow \infty .
$$

Since

$$
\frac{\partial^{2} \mathcal{H}_{0}}{\partial J_{1} \partial J_{2}}=0
$$

we have that

$$
\operatorname{det}\left(\frac{\partial^{2} \mathcal{H}_{0}}{\partial \hat{J}_{k}^{2}}\right)=\frac{\partial^{2} \mathcal{H}_{0}}{\partial \hat{J}_{1}^{2}} \frac{\partial^{2} \mathcal{H}_{0}}{\partial \hat{J}_{2}^{2}} \neq 0,
$$

so that eq.(78) is satisfied and condition 1 . in the statement of Theorem 2 is verified. Q.E.D.

We come now to the genericity condition, directly proceeding to perform a Fourier series expansion of the perturbation appearing in (60):

$$
\mathcal{H}_{1}=\mathrm{e}^{\alpha(\eta)-\beta(\eta)}=\sum_{m, n=-\infty}^{+\infty} h_{m n}\left(J_{1}, J_{2}\right) \mathrm{e}^{i\left(m \theta_{1}+n \theta_{2}\right)},
$$


where we have to use the solution of the unperturbed problem. The motion in the potential of Hamiltonian (60) with $E=0$ is given by

$$
\begin{aligned}
& \alpha(\eta)=2 \arcsin \left[\kappa_{1} \operatorname{sn}\left(\eta, \kappa_{1}\right)\right], \\
& \beta(\eta)=2 \arcsin \left[\kappa_{2} \operatorname{sn}\left(\eta, \kappa_{2}\right)\right],
\end{aligned}
$$

where $\operatorname{sn}(u)$ is the Jacobi sine-amplitude function. In this way, since $\kappa$ depends on $J$, the actions of the unperturbed problem enter in the expansion. On the same footing, the angles appearing in the Fourier expansion are just the canonical angle variables of the unperturbed problem, so that the harmonics $m \omega_{1}, n \omega_{2}$, with integer $m, n$, are multiples of the unperturbed frequencies.

We preliminarly assess the structure of the resonating set of frequencies that determine the Poincaré set. Using expressions (81) for the frequencies, we have that the resonance condition (76) can be written as

$$
\frac{q}{K\left(\kappa_{1}^{2}\right)}-\frac{p}{K\left(\kappa_{2}^{2}\right)}=0
$$

where, in view of (68) and (70),

$$
\kappa_{2}^{2}=1-C-\kappa_{1}^{2} .
$$

Choosing a value of the parameter $C$ in the interval (48) fixes the corresponding range of $\kappa_{1}^{2}$ as

$$
0 \leq \kappa_{1}^{2} \leq 1-C .
$$

Therefore, we can define a function

$$
R\left(\kappa_{1}^{2}\right)=\frac{q}{p} K\left(1-C-\kappa_{1}^{2}\right)-K\left(\kappa_{1}^{2}\right),
$$

which is continuous in the range (92) and, recalling (84), such that

$$
R(0)=\frac{q}{p} K(1-C)-\frac{\pi}{2}, \quad R(1-C)=\frac{\pi q}{2 p}-K(1-C) .
$$

Without loss of generality we have assumed $p \leq q$. Recalling again (84), we have that

$$
K(1-C)>\frac{\pi}{2}
$$

which implies $R(0)>0$. Moreover, if the inequality

$$
\frac{q}{p}<\frac{2}{\pi} K(1-C)
$$

is satisfied, we see that $R(1-C)<0$. Then we have that the function $R\left(\kappa_{1}^{2}\right)$ defined in the interval (92), by continuity, must necessarily vanish at a point of this interval. Observing that condition (90) can also be written in the form

$$
\frac{\frac{q}{p} K\left(1-C-\kappa_{1}^{2}\right)-K\left(\kappa_{1}^{2}\right)}{K\left(1-C-\kappa_{1}^{2}\right) K\left(\kappa_{1}^{2}\right)}=0
$$


we see that the point where $R\left(\kappa_{1}^{2}\right)$ vanishes is also the point where the resonance condition is satisfied.

Actually, it is straightforward to check that inequality (95) is not a limitation on the set of possible resonances, since dynamics automatically comply with it. In fact, recalling again (81) and 84), we see that both frequencies of the unperturbed libration motions must satisfy the constraints

$$
\frac{\pi}{2 K(1-C)}=\omega(1-C)<\omega<\omega(0)=1 .
$$

Therefore, the ratio of two frequencies is such that

$$
\frac{\omega_{2}}{\omega_{1}}<\frac{\omega(0)}{\omega(1-C)}=\frac{2}{\pi} K(1-C), \quad \forall C \in(0,1] .
$$

As a consequence we see that, for every pair of frequencies such that the resonance condition (76) is verified, inequality (95) is always satisfied. We conclude that, for every value of the parameter $C$ in the range (48), there is a whole dense set of resonances in the space of unperturbed frequencies. Each value of the root of the function $R\left(\kappa_{1}^{2}\right)$, say $\hat{\kappa}_{1}^{2}(C, q, p)$ with the corresponding value of $\hat{\kappa}_{2}^{2}$ given by (91), determine, via (69), the corresponding values of $\hat{J}_{1}$ and $\hat{J}_{2}$ of the Poincaré set.

At this point, we have the following:

Lemma 4: The Fourier expansion coefficients of perturbation 877) are nonvanishing on the resonance manifold specified by relation (76).

Proof: The Fourier coefficients are given by

$$
h_{m n}=A_{m} B_{n},
$$

where

$$
\begin{aligned}
& A_{m}=\frac{1}{T_{1}} \int_{0}^{T_{1}} \mathrm{e}^{\alpha(\eta)} \mathrm{e}^{-i m \omega_{1} \eta} d \eta, \\
& B_{n}=\frac{1}{T_{2}} \int_{0}^{T_{2}} \mathrm{e}^{-\beta(\eta)} \mathrm{e}^{-i n \omega_{2} \eta} d \eta,
\end{aligned}
$$

and, in a natural way,

$$
T_{k}=\frac{2 \pi}{\omega_{k}}, \quad k=1,2 .
$$

On the resonance manifold we have

$$
\frac{m}{n}=-\frac{q}{p},
$$

so that the simplest choice we can make is

$$
m=q, \quad n=-p .
$$


Let us separate real and imaginary parts in the Fourier coefficients of a generic resonance. The coefficient with $m=q$ is:

$$
A_{q}=\frac{1}{T_{1}} \int_{0}^{T_{1}} \mathrm{e}^{\alpha(\eta)}\left(\cos q \hat{\omega}_{1} \eta-i \sin q \hat{\omega}_{1} \eta\right) d \eta=C_{q}-i S_{q} .
$$

Using the resonance condition

$$
n \hat{\omega}_{2}=-p \hat{\omega}_{2}=-q \hat{\omega}_{1}
$$

the coefficient with $n=-p$ is:

$$
B_{-p}=\frac{q}{p T_{1}} \int_{0}^{\frac{p}{q} T_{1}} \mathrm{e}^{-\beta(\eta)}\left(\cos q \hat{\omega}_{1} \eta+i \sin q \hat{\omega}_{1} \eta\right) d \eta=C_{p}+i S_{p} .
$$

The Fourier coefficients on the resonance manifold are therefore

$$
h_{q,-p}=\left(C_{q} C_{p}+S_{q} S_{p}\right)+i\left(C_{q} S_{p}-C_{p} S_{q}\right) .
$$

We now proceed to evaluate above integrals, starting with the elimination of all cases in which some of them identically vanish. First of all, observing solutions (88 89), we realize that the functions $\alpha(\eta)$ and $\beta(\eta)$ are even over each half oscillation period and odd over the corresponding whole period. As a consequence, the exponentials appearing in the perturbation (87) are both even functions over the corresponding half period. On the same interval, $\cos q \hat{\omega}_{1} \eta$ (with $q$ odd) and $\sin q \hat{\omega}_{1} \eta$ (with $q$ even) are odd functions. Referring to the integrals in (104), by parity arguments, we can therefore state that:

$$
\begin{aligned}
& C_{q}=0 \text { for } q \text { odd, } \\
& S_{q}=0 \text { for } q \text { even. }
\end{aligned}
$$

The remaining integrals, namely $S_{q}$ with $q$ odd and $C_{q}$ with $q$ even, are surely non vanishing. For, take for instance $S_{q}$ when $q$ is odd. We have

$$
\begin{aligned}
T_{1} S_{q} & =\int_{0}^{\frac{1}{2} T_{1}} \mathrm{e}^{\alpha(\eta)} \sin q \hat{\omega}_{1} \eta d \eta+\int_{\frac{1}{2} T_{1}}^{T_{1}} \mathrm{e}^{\alpha(\eta)} \sin q \hat{\omega}_{1} \eta d \eta \\
& =\int_{0}^{\frac{1}{2} T_{1}} \mathrm{e}^{\alpha(\eta)} \sin q \hat{\omega}_{1} \eta d \eta-\int_{0}^{\frac{1}{2} T_{1}} \mathrm{e}^{-\alpha(\eta)} \sin q \hat{\omega}_{1} \eta d \eta,
\end{aligned}
$$

where, in the second step, we performed a translation $\eta \rightarrow \eta-T_{1} / 2$ and used the fact that

$$
\begin{aligned}
\alpha\left(\eta-T_{1} / 2\right) & =-\alpha(\eta) \\
\sin q \hat{\omega}_{1}\left(\eta-T_{1} / 2\right) & =-\sin q \hat{\omega}_{1} \eta .
\end{aligned}
$$

The statement follows because

$$
\mathrm{e}^{\alpha(\eta)} \neq \mathrm{e}^{-\alpha(\eta)}, \forall \eta \neq 0 .
$$


The same holds for $C_{q}$ when $q$ is even.

The integrals appearing in (105) are characterized by the integration interval which depends on the ratio $p / q$. However, the function $\exp (-\beta)$, which has the same parity property of $\exp (-\alpha)$, is "sampled" by trigonometric functions alternatively even and odd on each half interval. Using the same arguments as above we get

$$
\begin{aligned}
& C_{p}=0, \quad S_{p} \neq 0, \text { for } p \text { odd } \\
& S_{p}=0, \quad C_{p} \neq 0, \text { for } p \text { even. }
\end{aligned}
$$

Summarizing these results and comparing with expression (106), we get finally that the following coefficients

$$
\begin{aligned}
h_{q,-p} & =C_{q} C_{p} \text { for } q, p \text { even, } \\
h_{q,-p} & =i C_{q} S_{p} \text { for } q \text { even and } p \text { odd, } \\
h_{q,-p} & =-i S_{q} C_{p} \text { for } q \text { odd and } p \text { even, } \\
h_{q,-p} & =S_{q} S_{p} \text { for } q, p \text { odd },
\end{aligned}
$$

are non vanishing. This proves the lemma and shows that condition 2 . in the statement of theorem 2 is indeed satisfied. Q.E.D.

We remark that, according to the analysis already carried out by Poincaré (1892, sect.83), condition 2. can be somewhat relaxed. In particular, introducing the concept of "classes" in the resonance manifold, the first combination with both $q$ and $p$ even can be reduced to one of the others. However, this fact does not substantially modify the conclusions above.

With this body of results we can now enunciate our non-integrability statement applied to the original class of systems of section 3.1.

Theorem 5: The dynamical system generated by Hamiltonian,

$$
\mathcal{H}=\frac{1}{2}\left(p_{r}^{2}+\frac{p_{\vartheta}^{2}}{r^{2}}\right)+\frac{C-\sin \vartheta \cos (\ln r)}{r^{2}}=E,
$$

integrable at energy $E=0$, does not admit a conserved function

$$
I\left(p_{r}, p_{\vartheta}, r, \vartheta ; C\right)
$$

defined in the open set $-\epsilon_{1}<E<0$ and $0<E<\epsilon_{2}$, with small enough $\epsilon_{1,2}$, for every value of $C$ in the interval $(0,1]$.

Proof: From the discussion on regularization and dynamics at zero energy of sects. 2.3 and 2.4, it has been shown that the dynamics generated by Hamiltonian (119) is equivalent, a part a time reparametrization, to those generated by Hamiltonian (60). In particular, this applies to the conservation properties of phase-space functions: non-existence of a function $I\left(p_{\alpha}, p_{\beta}, \alpha, \beta ; E\right)$ which is an invariant, for any $E$, of Hamiltonian (60), implies non-existence of a function 
$I\left(p_{r}, p_{\vartheta}, r, \vartheta ; C\right)$ which is an invariant, for any $C$, of Hamiltonian (119). Hamiltonian (60) satisfies the conditions for the applicability of theorem 2. For, by lemma 3 we have that its unperturbed part is non degenerate and from lemma 4 we have that the perturbing function is generic in the Poincare set which is dense for every $0<C \leq 1$. The statement follows. Q.E.D.

We recall (see sect. 3.1) that Hamiltonian (119) supports bound motion in the energy range

$$
E_{\min }<E<E_{T}
$$

with $E_{\min }<0$ and $E_{T}>0$ and is separable at $E=0$. Theorem 5 proves non-integrability of these motions for small (positive or negative) values of the energy. However, from the remarks above, it is not possible to exclude the existence of isolated integrable case with non uniform invariants corresponding to some other fixed values of the energy.

\subsection{Existence of isolated periodic orbits}

Non-integrability of a perturbed Hamiltonian system manifests itself with the birth of alternately stable and unstable periodic orbits. Actually, their existence is an "obstruction" to the integrability and can be used as an argument to prove non-integrability when the conditions required by theorem 2 do not apply or are difficult to be checked. In our case, we can exploit the results already obtained to get informations about the nature of the periodic orbits ensuing from the perturbation. We follow Arnold, Kozlov and Neishtadt (1988, sect. 6.1) to show the existence of isolated periodic orbits and to determine their stability properties. For a recent application of this technique in celestial mechanics we refer to Diacu and Santoprete (2001).

We state, referring for the proof again to Poincaré (1892), the following

Theorem 6: Suppose that the following conditions are satisfied:

1. $\mathcal{H}_{0}$ is non-degenerate in $\hat{J}_{k}, k=1,2$, that is:

$$
\operatorname{det}\left(\frac{\partial^{2} \mathcal{H}_{0}}{\partial \hat{J}_{k}^{2}}\right) \neq 0
$$

2. for some $\lambda=\lambda^{\star}$, the average perturbation, defined as

$$
\overline{\mathcal{H}}_{1}\left(\hat{J}_{1}, \hat{J}_{2}, \lambda\right)=\frac{1}{T_{\eta}} \int_{0}^{T_{\eta}} \mathcal{H}_{1}\left(\hat{J}_{1}, \hat{J}_{2}, \hat{\omega}_{1} \eta+\lambda, \hat{\omega}_{2} \eta\right) d \eta
$$

is such that

$$
\frac{\partial \overline{\mathcal{H}}_{1}}{\partial \lambda}=0, \quad \frac{\partial^{2} \overline{\mathcal{H}}_{1}}{\partial \lambda^{2}} \neq 0
$$

and the perturbing function

$$
\mathcal{H}_{1}=\mathcal{H}_{1}\left(\hat{J}_{1}, \hat{J}_{2}, \hat{\omega}_{1} \eta+\lambda, \hat{\omega}_{2} \eta\right)
$$


where $\lambda$ is the phase shift of the resonating motions, is periodic in $\eta$ with period $T_{\eta}$.

Then, for small $\epsilon$, the perturbed system has a periodic solution with period $T_{\eta}(\epsilon)$ such that $T_{\eta}(0)=T_{\eta}$ and with characteristic exponents $\pm \alpha$ which can be expressed as power series in the form

$$
\alpha=\alpha_{1} \sqrt{\epsilon}+\alpha_{2} \epsilon+\alpha_{3} \epsilon \sqrt{\epsilon}+\operatorname{dots},
$$

where the coefficients are given by the relations

$$
\hat{\omega}_{1}^{2} \alpha_{1}^{2}=\frac{\partial^{2} \overline{\mathcal{H}}_{1}\left(\lambda^{\star}\right)}{\partial \lambda^{2}}\left(\hat{\omega}_{1}^{2} \frac{\partial^{2} \mathcal{H}_{0}}{\partial \hat{J}_{1}^{2}}-2 \hat{\omega}_{1} \hat{\omega}_{2} \frac{\partial^{2} \mathcal{H}_{0}}{\partial \hat{J}_{1} \partial \hat{J}_{2}}+\hat{\omega}_{2}^{2} \frac{\partial^{2} \mathcal{H}_{0}}{\partial \hat{J}_{2}^{2}}\right) .
$$

We can actually prove that system (61) meets the requirement of theorem 6. For, condition 1 . is a straightforward application of lemma 3 to the Poincaré set. Condition 2. derives applying the results of lemma 4. Taking into account the phase shift, the Fourier expansion of the perturbation can now be written in the form

$$
\mathcal{H}_{1}\left(J_{1}, J_{2}, \eta, \lambda\right)=\sum_{m, n} \tilde{A}_{m}(\lambda) B_{n} \mathrm{e}^{i\left(m \omega_{1}+n \omega_{2}\right) \eta}
$$

where

$$
\tilde{A}_{m}(\lambda)=\frac{1}{T_{1}} \mathrm{e}^{i m \omega_{1} \lambda} \int_{0}^{T_{1}} \mathrm{e}^{\left[\alpha(\tau)-i m \omega_{1} \tau\right]} d \tau=\mathrm{e}^{i m \omega_{1} \lambda} A_{m}
$$

and

$$
\tau=\eta+\lambda,
$$

In order to verify condition 2. we have to perform the average 123) of $\mathcal{H}_{1}$. Using the expansion (128), we realize that the only $\lambda$ dependent terms surviving the averaging process, are just those corresponding to resonances of the form (76), that is

$$
\mathcal{H}_{1}\left(\hat{J}_{1}, \hat{J}_{2}, \eta, \lambda\right)=\sum_{m, n} \tilde{A}_{m}(\lambda) B_{n} \mathrm{e}^{i\left(m+n \frac{q}{p}\right) \hat{\omega}_{1} \eta} .
$$

Therefore, after averaging, we get terms of the form

$$
\overline{\mathcal{H}}_{1}\left(\hat{J}_{1}, \hat{J}_{2}, \lambda\right)=2 \operatorname{Re}\left\{\mathrm{e}^{i q \hat{\omega}_{1} \lambda} A_{q} B_{-p}\right\} .
$$

With the same notation used in the proof of lemma 4 , first and second derivative of $\overline{\mathcal{H}}_{1}\left(\hat{J}_{1}, \hat{J}_{2}, \lambda\right)$ with respect to $\lambda$ are:

$$
\begin{aligned}
\frac{\partial \overline{\mathcal{H}}_{1}}{\partial \lambda} & =2 \operatorname{Re}\left\{i q \hat{\omega}_{1}\left[\mathrm{e}^{i q \hat{\omega}_{1} \lambda}\left(C_{q}+i S_{q}\right)\left(C_{p}+i S_{p}\right)\right]\right\}, \\
\frac{\partial^{2} \overline{\mathcal{H}}_{1}}{\partial \lambda^{2}} & =2 \operatorname{Re}\left\{-q^{2} \hat{\omega}_{1}^{2}\left[\mathrm{e}^{i q \hat{\omega}_{1} \lambda}\left(C_{q}+i S_{q}\right)\left(C_{p}+i S_{p}\right)\right]\right\} .
\end{aligned}
$$

Therefore, condition 2. is satisfied if a $\lambda=\lambda^{\star}$ exists such that the imaginary part of the term in the square bracket vanishes with a non vanishing real part. The imaginary part is

$$
\cos q \hat{\omega}_{1} \lambda\left(C_{q} S_{p}+C_{p} S_{q}\right)+\sin q \hat{\omega}_{1} \lambda\left(C_{q} C_{p}-S_{q} S_{p}\right) .
$$


This expression vanishes if $\lambda$ takes the value

$$
\lambda^{\star}=\frac{1}{q \hat{\omega}_{1}} \arctan \frac{C_{q} S_{p}+C_{p} S_{q}}{S_{q} S_{p}-C_{q} C_{p}} .
$$

Correspondingly, we have:

$$
\left.\frac{\partial^{2} \overline{\mathcal{H}}_{1}}{\partial \lambda^{2}}\right|_{\lambda=\lambda^{\star}}=-2 q^{2} \hat{\omega}_{1}^{2} \cos q \hat{\omega}_{1} \lambda^{\star}\left[C_{q}\left(C_{p}+S_{p}\right)+S_{q}\left(C_{p}-S_{p}\right)\right] .
$$

Using the results summarized in (107) and (113), if $n$ denotes an arbitrary integer, we get that the critical shifts are either

$$
\lambda^{\star}=\frac{n \pi}{q \hat{\omega}_{1}}
$$

in case of an odd-odd or even-even $q, p$ combination, or

$$
\lambda^{\star}=\frac{n \pi}{2 q \hat{\omega}_{1}}
$$

in case of an even-odd or odd-even $q, p$ combination.

As a simple application, let us take the simplest case, that is

$$
p=q=1, \quad \hat{\omega}_{1}=\hat{\omega}_{2},
$$

so that the two unperturbed motions are identical but for the phase shift. From (137) we then have

$$
\lambda^{\star}=\frac{n \pi}{\hat{\omega}_{1}} .
$$

In particular,

$$
\lambda^{\star}=0 \rightarrow \frac{\partial^{2} \overline{\mathcal{H}}_{1}}{\partial \lambda^{2}}=\hat{\omega}_{1}^{2} S_{q=1}^{2}>0,
$$

so that, for the zero phase shift corresponding to the unperturbed periodic orbit (173), the leading order term in the expansion (126) of the characteristic exponent is a real number. This can be seen using eq. (127) with the result (139) above and with the round bracket that is positive in virtue of (83) and (85). Therefore, the characteristic exponents are real if $E<0$ and pure imaginary if $E>0$. The periodic orbit is then unstable in the former case and stable in the second. This change in the nature of a periodic orbit on varying the energy is another typical feature of non-integrable dynamics.

\section{Conclusions}

The main points which we have focused on in the present paper can be summarized as follows:

- There exist wide classes of natural conservative mechanical systems with integrable (actually separable) dynamics at energy equal to zero. 
- In the general case, the dynamics of the system is non-integrable at energies different from zero.

- The technique exploited here to construct these systems, which is based on a generic conformal transformation, offers the possibility of characterizing many features of the non-integrable dynamics.

In particular, we have seen how the structure of the transformed Hamiltonian naturally allows a perturbative approach to investigate the departure from the integrable regime. Standard tools of analytical mechanics, like the Poincaré methods, can be applied more easily than in the usual setting, to get analytical proofs of non-integrability.

A natural continuation of this work is to explore the character of other systems constructed by means of other conformal transformations or even of other potentials in the classes introduced above. In particular, we have limited the analysis to systems admitting bound motion, but in the class of systems studied above, it is easy to construct potentials allowing the coexistence of limited and unlimited motions. In such systems, dynamics are characterized by a transition to chaotic scattering with possible applications in celestial mechanics, quantum mechanics and general relativity.

\section{Acknowledgements}

We acknowledge the remarks of the referee, Dr. A. Albouy, that played a substantial role in producing a clearer presentation of the results.

\section{References}

[1] Abraham, R. and Marsden, J. E.: 1978, Foundations of mechanics, Benjamin Cummings

[2] Arnold, V. I.: 1978, Mathematical methods of classical mechanics, SpringerVerlag

[3] Arnold, V. I. Kozlov V. V. and Neishtadt, A. I.: 1988, Mathematical aspects of classical and celestial mechanics, Dynamical Systems III, SpringerVerlag

[4] Benenti, S.: 1997, Intrinsic characterization of the variable separation in the Hamilton-Jacobi equation, J. Math. Phys., 38, 6578-6602

[5] Boccaletti, D. and Pucacco, G.: 1996, Theory of Orbits: Vol. 1, SpringerVerlag

[6] Darboux, G.: 1901, Sur un problème de mécanique, Archives Néerlandaises (ii), VI, 371-377

[7] Diacu F. and Santoprete M.: 2001, Nonintegrability and chaos in the anisotropic Manev problem, Physica D, 156, 39-52 
[8] Giorgilli, A.: 2002, Notes on Exponential Stability of Hamiltonian Systems, Centro di Ricerca Matematica E. De Giorgi, Pisa

[9] Hall, L. S.: 1983, A theory of exact and approximate configurational invariants, Physica D, 8, 90-116

[10] Hietarinta, J.: 1987, Direct methods for the search of the second invariant, Physics Reports, 147, 87-153

[11] Karlovini, M. and Rosquist, K.: 2000, A unified treatment of cubic invariants at fixed and arbitrary energy, J. Math. Phys., 41, 370-384

[12] Karlovini, M. Pucacco, G. Rosquist, K. and Samuelsson, L.: 2002, A unified treatment of quartic invariants at fixed and arbitrary energy, J. Math. Phys., 43, 4041-4059

[13] Kozlov, V. V.: 1991, Symmetries, Topology and Resonances in Hamiltonian Mechanics, Springer-Verlag

[14] Lanczos, C.: 1986, The variational principles of mechanics, Dover

[15] Levi-Civita, T.: 1956, Opere Matematiche, Vol. 2, Bologna

[16] Markushevitch, A. I.: 1983, The Theory of Analytic Functions: a brief course, MIR

[17] Nakagawa, K. and Yoshida, H.: 2001, A list of all integrable 2D homogeneous polynomial potentials with a polynomial integral of order at most 4 in the momenta, J. Phys. A, 34, 8611-8630

[18] Poincaré, H.: 1892, Les Méthodes Nouvelles de la Mécanique Céleste, Vol. I, Gauthier-Villars

[19] Rosquist, K. and Pucacco, G.: 1995, Invariants at arbitrary and fixed energy: a unified geometric approach, J. Phys. A, 28, 3235-3252 (RP)

[20] Sarlet, W. Leach, P. G. L. and Cantrijn, F.: 1985, First integrals versus configurational invariants and a weak form of complete integrability, Physica $D, \mathbf{1 7}, 87-98$

[21] Sundman, K. F.: 1912, Mémoire sur le probléme de trois corps, Acta Mathematica, 36, 105-179

[22] Tsiganov, A. V.: 2000, Canonical transformations of the extended phase space, Toda lattices and Stäckel family of integrable systems, J. Phys. A, 33, 4169-4182

[23] Whittaker, E. T.: 1937, A Treatise on the Analytical Dynamics of Particles and Rigid Bodies, 4th ed., Cambridge Univ. Press 